\title{
Continuous and Discrete (Classical) Heisenberg Spin Chain Revised ${ }^{\star}$
}

\author{
Orlando RAGNISCO and Federico ZULLO
}

Dipartimento di Fisica, Università di Roma Tre and Istituto Nazionale di Fisica Nucleare Sezione di Roma Tre, Via Vasca Navale 84, I-00146 Roma, Italy

E-mail: ragnisco@fis.uniroma3.it,federico.zullo@virgilio.it

Received December 29, 2006; Published online February 26, 2007

Original article is available at http://www.emis.de/journals/SIGMA/2007/033/

\begin{abstract}
Most of the work done in the past on the integrability structure of the Classical Heisenberg Spin Chain (CHSC) has been devoted to studying the $s u(2)$ case, both at the continuous and at the discrete level. In this paper we address the problem of constructing integrable generalized "Spin Chains" models, where the relevant field variable is represented by a $N \times N$ matrix whose eigenvalues are the $N^{\text {th }}$ roots of unity. To the best of our knowledge, such an extension has never been systematically pursued. In this paper, at first we obtain the continuous $N \times N$ generalization of the CHSC through the reduction technique for Poisson-Nijenhuis manifolds, and exhibit some explicit, and hopefully interesting, examples for $3 \times 3$ and $4 \times 4$ matrices; then, we discuss the much more difficult discrete case, where a few partial new results are derived and a conjecture is made for the general case.
\end{abstract}

Key words: integrable systems; Heisenberg chain; Poisson-Nijenhuis manifolds; geometric reduction; $R$-matrix; modified Yang-Baxter

2000 Mathematics Subject Classification: 37K05; 37K10

\section{Introduction}

The Heisenberg ferromagnet has been one of the first integrable models investigated and solved in the framework of the Inverse Scattering Method, both at a classical $[1,2,3]$ and at a quantum level. In particular, at the quantum level, through the Quantum Inverse Scattering Method $[4,5]$, the original Bethe solution [6] has been rediscovered and generalized, and the solution of the isotropic one-dimensional spin chain was just the starting example in a list of a number of outstanding achievements obtained in this field in the 80's and in the 90's of the last century. The scope of the present contribution is twofold. On one hand, in Section 2 the geometric approach introduced in the field of infinite dimensional integrable systems by Magri and coll. [7, 9,8] will be used to extend to $N \times N$ matrices the old results on the $S U(2)$ classical Heisenberg chain obtained about 20 years ago; accordingly, new hierarchies of integrable evolution equations will be derived, and a few explicit examples will be shown, which might have some relevance for studying classical analogs of the so-called Potts model $[10,11,12]$. On the other hand, in Section 3 the intriguing issues concerning the lattice version of the model $[2,13]$ will be reexamined, a few additional results in the $2 \times 2$ case will be given, and finally a conjecture concerning the general $N \times N$ case will be formulated.

${ }^{\star}$ This paper is a contribution to the Proceedings of the Workshop on Geometric Aspects of Integrable Systems (July 17-19, 2006, University of Coimbra, Portugal). The full collection is available at http://www.emis.de/journals/SIGMA/Coimbra2006.html 


\section{The classical Heisenberg chain on the line for $N \times N$ matrices}

Let us first recall the basic notions and results underlying the integrability of the classical Heisenberg ferromagnet in the usual $s u(2)$ setting. As a starting point, we will adopt the simplest point of view, where an integrable (matrix) system in $1+1$ continuous dimensions is regarded as the compatibility condition for two linear differential (matrix) systems, defining the so called associated Lax pair:

$$
\begin{aligned}
& \psi_{x}=\lambda S \psi, \\
& \psi_{t}=V \psi .
\end{aligned}
$$

In (2.1), (2.2) $\psi, S, V$ are $n \times n$ matrix valued functions of the $(x, t)$ coordinates, $S$ is the field variable appearing linearly in the so-called principal spectral problem, $V$ has an additional (rational) dependence on a complex parameter $\lambda$, denoted as the spectral parameter. It would be natural to look at $\psi$ as an element of a Lie group, and at $S, V$ as elements of the associated Lie algebra. More correctly, due to the extra dependence on $\lambda$, we should speak of "loop" Groups and Algebras. However, in the sequel we will always assume $S$ to belong to the open set of the invertible elements of the unital associative algebra of the $N \times N$ matrices with (generally) distinct eigenvalues.

The compatibility condition of the above linear system $(2.1),(2.2)$ reads:

$$
S_{t}=\frac{1}{\lambda} V_{x}+[V, S]
$$

which is also known as the zero-curvature condition for the connection given by the one form:

$$
\alpha=\lambda S d x-V d t .
$$

Actually, in the very simple example under scrutiny, the compatibility condition (2.3) amounts to the compatibility (i.e. vanishing of the Schouten bracket) of the two Poisson tensors:

$$
\mathcal{P} V=[V, S], \quad \mathcal{Q} V=V_{x}
$$

which then define the bi-Hamiltonian pair $\mathcal{P}, \mathcal{Q}$.

As is well known from [7, 9, 8], the Poisson tensors $\mathcal{P}, \mathcal{Q}$ are linear operators from the cotangent bundle $T^{*} M$ to the tangent bundle $T M$ to our configuration manifold, which is just an affine hyperplane to the linear space of matrix-valued $C^{\infty}$ functions of the real variable $x$ fulfilling homogeneous boundary conditions. The duality form between $T M$ and $T^{*} M$ is the usual trace form $\langle\phi, \alpha\rangle:=\int_{-\infty}^{\infty} \operatorname{Tr}(\phi \alpha) d x$. As $\mathcal{Q}$ is invertible, we can introduce on $T M$ the tensor (Recursion operator):

$$
\mathcal{N}:=\mathcal{P} \mathcal{Q}^{-1} \Rightarrow \phi^{\prime}\left(:=S_{t^{\prime}}\right)=\left[S, \int^{x} \phi\left(:=S_{t}\right)\right]
$$

that has zero Nijenhuis torsion (whence the name "Nijhenhuis tensor" [14]) and gives rise to a hierarchy of commuting flows. Under the above conditions we will say that $M$ is equipped with a $\mathcal{P N}$ structure.

In order to construct a hierarchy of integrable evolution equations, we have to choose a submanifold $M^{\prime} \subset M$ such that the Nijenhuis tensor has maximal rank on it. As shown in [8] we can achieve this goal by going on restricting the action of $\mathcal{N}$ to smaller and smaller submanifolds as far as the structure is preserved. In particular, it is always possible to restrict $\mathcal{N}$ on a characteristic leaf of $\operatorname{Im} \mathcal{Q}$, and then again on a leaf of the distribution generated by $\operatorname{Im} \mathcal{N}^{r}$, 
where $r$ (assumed to be finite) is the Riesz index of $\mathcal{N}$ [15]. As in our case $\mathcal{Q}$ is invertible, the only relevant restriction is on

$$
\operatorname{Im} \mathcal{N}=\operatorname{Im} \mathcal{P}\{\phi: \phi=[S, \alpha] \text { for some } \alpha\} .
$$

It can be readily seen that $\operatorname{Im} \mathcal{N}=\operatorname{Im} \mathcal{N}^{k}, \operatorname{Ker} \mathcal{N}=\operatorname{Ker} \mathcal{N}^{k}, \forall k>1$. Hence $r=1$ and then we can restrict the $\mathcal{P} \mathcal{N}$ structure on a characteristic leaf of the distribution (2.5):

$$
T M^{\prime}=\left\{\phi: \operatorname{Tr} S^{k} \phi=0\right\} \quad(k=0, \ldots, N-1)
$$

yielding the symplectic leaves:

$$
\operatorname{Tr} S^{k}=d_{k}
$$

The hierarchy obtained by acting with $\mathcal{N}(2.4)$ on the starting symmetry $[S, C], C$ being an arbitrary constant matrix, (the so-called "rotational flow") consists however of nonlocal equations. As it was already shown in [8] for $2 \times 2$ matrices, the way to get local equations is then to invert $\mathcal{N}$, which is obviously possible thanks to the above restriction.

In the following, for the sake of simplicity, we will simply denote by $\mathcal{N}^{(-1)}$ the inversion of the restriction of $\mathcal{N}$ on $(2.6)$.

The most important case, and the best studied one, is when $S \in s u(2)$, namely it is a $2 \times 2$ Hermitean traceless matrix:

$$
S=\vec{S} \cdot \hat{\sigma}, \quad \vec{S} \cdot \vec{S}=1, \quad \hat{\sigma}=\left(\sigma_{1}, \sigma_{2}, \sigma_{3}\right) .
$$

Finding $\mathcal{N}^{-1}$ is a rather easy task. Indeed, from

$$
\phi^{\prime}=\left[S, \int^{x} \phi\right]
$$

one gets

$$
\phi=\frac{1}{4}\left[S, \phi^{\prime}\right]_{x}+\left(c_{0} I+c_{1} S\right)_{x}
$$

where the second term in the above formula comes from $\operatorname{Ker}[S, \cdot]$. Whence, imposing $\phi$ to be tangent to the symplectic leaves (2.6), we derive:

$$
c_{0}=0, \quad c_{1}=-\frac{1}{8} \int^{x} \operatorname{tr}\left[S, S_{x}\right] \phi
$$

so that:

$$
\phi:=\mathcal{N}^{(-1)} \phi^{\prime}=\frac{1}{4}\left[S, \phi^{\prime}\right]_{x}-\frac{1}{8} S \int^{x} \operatorname{tr}\left[S, S_{x}\right] \phi .
$$

From (2.7) one gets a local hierarchy [8], whose first members are:

$$
\phi_{0}=0, \quad \phi_{-1}=S_{x}, \quad \phi_{-2}=\left[S, S_{x x}\right], \quad \ldots
$$

In particular the HSC Hamiltonian flow is $\phi_{-2}=\left[S, S_{x x}\right]$, namely:

$$
S_{t}=\left[S, S_{x x}\right]=[S, \nabla \mathcal{H}] \quad \Rightarrow \quad \mathcal{H}=\frac{1}{2} \int_{-\infty}^{+\infty} \operatorname{Tr}\left(S_{x}\right)^{2}
$$

The novel result contained in this section is the explicit form of $\mathcal{N}^{(-1)}$, and of the associated local evolution equations and Hamiltonian functionals for $N \times N$ matrices s.t. $S^{N}=I$, which 
we have denoted as $H S C$ at the $N^{\text {th }}$ roots of unity. The derivation is quite simple. Indeed, from the very definition of the Nijhenhuis tensor (2.4) one easily gets:

$$
\phi=\frac{1}{2 N} \frac{\partial}{\partial x}\left(\sum_{l=1}^{N-1} l\left[S^{l} \phi^{\prime} S^{N-l-1}-S^{N-l-1} \phi^{\prime} S^{l}\right]+\sum_{l=0}^{N-1} c_{l} S^{l}\right) .
$$

The conditions:

$$
\operatorname{Tr} S^{k} \phi=0
$$

that ensure $\phi$ to be tangent to (2.6), allow to determine $c_{k}$ through quadratures, up to constant integration factors:

$$
\left(c_{k}\right)_{x}=-\frac{2}{N} \operatorname{Tr} \sum_{l=1}^{N-1} l S^{n-l-1} \phi^{\prime} S^{l}\left(S^{N-k}\right)_{x} .
$$

For a given $N$, the hierarchy of evolution equations splits into $N-1$ sub-hierarchies, with starting symmetries $\left(S^{k}\right)_{x}(k=1, \ldots, N-1)$. In the following, we provide the explicit form of the first members of the hierarchy for $N=3$ and $N=4$.

Example 1. $N=3$. There are two families of local flows, with starting points:

$$
\phi_{1}^{(1)}=S_{x}, \quad \phi_{1}^{(2)}=\left(S_{x}\right)^{2}
$$

whence the first few members of simpler family read:

$$
\phi_{2}^{(1)}=\left[S^{2}, S_{x}\right]_{x}, \quad \phi_{3}^{(1)}=\left[S^{2},\left[S^{2}, S_{x}\right]_{x}\right]_{x}, \quad \ldots
$$

The Hamiltonian $\mathcal{H}_{2}^{(1)}$ reads (up to constant factors):

$$
\mathcal{H}_{2}^{(1)}=\int_{-\infty}^{+\infty} \operatorname{Tr} S\left(S_{x}\right)^{2} .
$$

Example 2. $N=4$. There are three families of local flows, with starting points:

$$
\phi_{1}^{(1)}=S_{x}, \quad \phi_{1}^{(2)}=\left(S_{x}\right)^{2}, \quad \phi_{1}^{(3)}=\left(S_{x}\right)^{3}
$$

whence the next flow in the simplest family reads:

$$
\phi_{2}^{(1)}=3\left[S^{3}, S_{x}\right]_{x}+\left[S, S S_{x} S\right]_{x},
$$

while the associated Hamiltonian functional $\mathcal{H}_{2}^{(1)}$ is given by:

$$
\mathcal{H}_{2}^{(1)}=\int_{-\infty}^{+\infty} \operatorname{Tr}\left(\left(S_{x}\right)^{2}-\frac{1}{8}\left(S S_{x}\right)^{2}\right)
$$

Remark 1. The flow (2.8) is obviously consistent with the restriction $S^{N}=I \forall N$. However, it belongs to the integrable hierarchies only for $N=2$. In fact, the Lie derivative of the Nijenhuis tensor (2.4) in the direction of $\psi:=\left[S, S_{x x}\right]$ on an arbitrary vector $\phi$ can be calculated from the formula:

$$
\mathcal{L}_{\psi}(\mathcal{N} \phi)=\left(\mathcal{L}_{\psi}(\mathcal{N})(\phi)+\mathcal{N} \mathcal{L}_{\psi} \phi\right.
$$

whence, with the proper replacements:

$$
\left(\mathcal{L}_{\psi}(\mathcal{N})(\phi)=3\left[S,\left[\phi, S_{x}\right]\right]\right.
$$

From (2.9) we readily see that the Lie derivative of the Nijenhuis tensor in the direction (2.8) vanishes only for the subhierarchies starting with $\phi_{1}^{(1)}=S_{x}$. Hence, for $N$ larger than 2 , the flow (2.8), though being Hamiltonian and possessing infinitely many conserved quantities (and commuting symmetries) is certainly not completely integrable. 


\section{The discrete case: some results and a conjecture}

In contrast with the continuous case, we cannot give any new result for arbitrary $N \times N$ matrices. However, before focussing on the well known $2 \times 2$ case, we will write down the compatibility scheme and recall the abstract $\mathcal{P} \mathcal{N}$ structure in the differential-difference setting, as it has been elucidated in the past by various authors $[13,17,18]$. The two relevant linear problems read:

$$
\begin{aligned}
& \psi_{m+1}=\left(I+\lambda S_{m}\right) \psi_{m}, \\
& \psi_{m, t}=V_{m}(\lambda) \psi_{m} .
\end{aligned}
$$

In (3.1) $\psi, S, V$ are $N \times N$ matrices, depending on a discrete variable $m \in \mathbb{Z}$, on a continuous variable $t \in \mathbb{R}$ and on a complex parameter $\lambda$. It has been shown $[13,17,18]$ that the compatibility of the above linear problems yields the $\mathcal{P} \mathcal{N}$ structure:

$$
\begin{aligned}
\phi & :=\mathcal{P} \alpha=[S, \alpha], \\
\phi^{\prime} & :=N \phi=\frac{1}{2}[S,(\mathbf{R} \phi)]+\frac{1}{2}\{S \phi\},
\end{aligned}
$$

where:

$$
\mathbf{R}=\frac{\mathcal{E}+1}{\mathcal{E}-1}, \quad(\mathcal{E} \phi)_{m}:=\phi_{m+1} .
$$

We note that $\mathbf{R}$ is the so-called ILW operator, satisfying the modified Yang-Baxter equation in the algebra of shift operators [17].

Remark 2. Notice that now $\operatorname{Im} \mathcal{N}$ is larger than $\operatorname{Im} \mathcal{P}$; however $\operatorname{Im} \mathcal{P}$ is still an invariant submanifold for $\operatorname{Im} \mathcal{N}$, namely $\operatorname{Tr} S^{l} \phi=0$ implies $\operatorname{Tr} S^{l} \mathcal{N} \phi=0 \forall l$. Indeed, from (3.2) it follows the recurrence relation $\operatorname{Tr} S^{l} \mathcal{N} \phi=\operatorname{Tr} S^{l+1} \phi$, entailing $\operatorname{Tr}\left[\mathcal{N}^{N}-\mathcal{I}\right] \phi=0=\operatorname{Tr} S^{l}\left[\mathcal{N}^{N}-\mathcal{I}\right] \phi, \forall l$.

As we mentioned before, to the best of our knowledge explicit evolution equations can be found in the literature only for $2 \times 2$ matrices. They are for instance reported in the fundamental reference [2], where the corresponding "Lax pair" (3.1) is also shown. As it was first pointed out in [13], both the Discrete Heisenberg (DH) ferromagnet and the Discrete Translational (DT) flow belong to the kernel of $\mathcal{N}^{2}-\mathcal{I}$, which is in fact the linear span of them. They read respectively:

$$
\begin{aligned}
& S_{m, t}=\left[S_{m}, \beta_{m} S_{m+1}+\beta_{m-1} S_{m-1}\right], \\
& S_{m, \tau}=\beta_{m}\left(S_{m}+S_{m+1}\right)-\beta_{m-1}\left(S_{m}+S_{m-1}\right),
\end{aligned}
$$

where $\beta_{m}:=\frac{1}{\operatorname{Tr}\left(I+S_{m} S_{m+1}\right)}$. Each of the above two equations can be derived from the other by applying the recursion operator $\mathcal{N}$.

The above results, and Remark 2 strongly suggest the following

Conjecture. For $N \times N$ matrices obeying the cyclic constraint $S^{N}=I$, the local evolution equations span $\operatorname{Ker}\left(\mathcal{N}^{N}-\mathcal{I}\right)$; hence, starting from any of them, the others can be obtained by applying subsequently $\mathcal{N}$. In other words, we can always find in $\operatorname{Ker}\left(\mathcal{N}^{N}-\mathcal{I}\right)$ a cyclic basis $\phi^{(r)}$ such that: $\phi^{(r+1)}=\mathcal{N} \phi^{(r)}, \phi^{(r+N)}=\phi^{(r)}$.

\section{Acknowledgements}

O. Ragnisco would like to thank the organizers of the Geomis workshop, and in particular Joana Nunes da Costa, for the admirable work they have done in preparing and directing the meeting, and for their kind and warm hospitality in Coimbra. Also, O.R. acknowledges illuminating discussions with his long-time colleague and friend Franco Magri. 


\section{References}

[1] Takhtajan L.A., Integration of the continuous Heisenberg spin chain through the inverse scattering method, Phys. Lett. A 64 (1977), 235-237.

[2] Faddeev L.D., Takhtajan L.A., Hamiltonian methods in the theory of solitons, Springer Series in Soviet Mathematics, Berlin, 1987.

[3] Orphanidis S.J., SU $(N)$ Heisenberg spin chain, Phys. Lett. A 75 (1980), 304-306.

[4] Kulish P.P., Sklyanin E.K., Quantum spectral transform method, recent developments, in Integrable Quantum Field Theories, Lecture Notes in Physics, Vol. 151, Editors J. Hietarinta and C. Montonen, 1982, $61-119$

[5] Sklyanin E.K., Quantum inverse scattering method. Selected topics, in Quantum Groups and Quantum Integrable Systems, Editor M.L. Ge, Nankai Lectures in Mathematical Physics, World Scientific, Singapore, 1992, 63-97, hep-th/9211111.

[6] Bethe H.A., Zur Theorie der Metalle I. Eigenwerte und Eigenfunktionen der linearen Atomkette, Z. Phys. 71 (1931), 205-226.

[7] Magri F., Morosi C., A geometrical characterization of integrable Hamiltonian systems through the theory of Poisson-Nijenhuis manifolds, Quaderno di matematica S19, Dipartimento di matematica, Università di Milano, 1984.

[8] Magri F., Morosi C., Ragnisco O., Reduction techniques for infinite-dimensional Hamiltonian systems: some ideas and applications, Comm. Math. Phys. 99 (1985), 115-140.

[9] Kosmann-Schwarzbach Y., Magri F., Poisson-Nijenhuis structures, Ann. Inst. H. Poincaré, Phys. Theor. 53 (1990), 35-81.

[10] Potts R., Some generalized order-disorder transformations, Proc. Camb. Phil. Soc. 48 (1952), $106-109$.

[11] Wu F.Y., The Potts model, Rev. Modern Phys. 54 (1982), 235-268.

[12] Hartmann A.K., Calculation of partition functions by measuring component distributions, Phys. Rev. Lett. 94 (2005), 050601, 4 pages, cond-mat/0410583.

[13] Ragnisco O., Santini P.M., A unified algebraic approach to integral and discrete evolution equations, Inverse Problems 6 (1990), 441-452.

[14] Nijenhuis A., Connection-free differential geometry, in Proc. Conf. Differential Geometry and Applications, (August 28 - September 1, 1995, Masaryk University, Brno, Czech Republic), Editors J. Janyska, I. Kolar and J. Slovak, Masaryk University Brno, 1996, 171-190.

[15] Kreyszig E., Introductory functional analysis with applications, Wiley Classic Library, 1989.

[16] Morosi C., The R-matrix theory and the reduction of Poisson manifolds J. Math. Phys. 33 (1992), 941-952.

[17] Morosi C., Tondo G., Yang-Baxter equations and intermediate long wave hierarchies, Comm. Math. Phys 122 (1989), 91-103.

[18] Morosi C., Tondo G., Some remarks on the bi-Hamiltonian structure of integral and discrete evolution equations, Inverse Problems 6 (1990), 557-566. 